\section{ON THE TREATMENT OF DEFECTIVE UTERINE INVOLUTION.}

\section{BY EDWARD JOHN TILT, M.D.}

IF it was right to consider defective uterine involution as, to a great extent, the workshop of uterine pathology, the matter in hand is important, for there is evident danger of some disease setting up, in a womb that remains much larger than it ought to be several months after delivery.

There is firstly to be considered the right performance of what should be a natural function; so pregnancy and labour should be managed in order to ensure the maintenance of sufficient reserve-force when the system shall be called upon to reconstruct the womb; and I think Dr. Bassett* is right in urging that many women want more medical treatment than they usually get during pregnancy-that they want tonics, and, above all, iron. It is also clear that, whatever prolongs labour and exhausts strength augments the chances of defective involution. It is, moreover, fair to conclude that too long-continued contraction of the uterine muscle leaves it half paralysed after delivery, and that its overstrained muscular fibres can no more properly contract, than can the muscular fibres of the over-distended bladder after the urine is removed. I therefore look on the present tendency to shorten labour by instrumental interference, so goon as it can be safely done, as well calculated to preven involution becoming defective. Those who object to medical treatment during pregnancy, and to the expediting of labour, on the plea of interference with natural functions, should bear in mind that we have to do with women considerably modified by hereditary tendencies, and by the bad hygienic habits that always wait on a high state of civilisation. Some of the women we attend have never done a hard day's work till labour pains set in.

The giving a full dose of ergot as the head emerges, and Credés method of removing the placenta, tends to prevent post-partum hæmorrhage, and gives the womb a proper shape, by making its muscular fibres fully contract in the right direction ; for however firmly it may at first contract, the womb soon relaxes ; for it is in the midst of the fastdecaying elements of worn-out muscular tissue, that a new womb is built up, just as in dissolving views we see new forms arise in the very midst of structures that rapidly melt away. It can therefore be of no use to apply firm pressure to the womb after the first few hours of delivery and a bandage then should have no other object than to give the abdominal viscera that support they cannot get from relaxed abdominal walls. Should there be a previous history of some uterine distortion, attention should be given to the patient's posture in bed. She should be told to lie as much as possible on her back if the womb had previously been anteverted or anteflexed; and, on the contrary, to lie as much as possible on the face and stomach if, as is more frequently the case, the womb had been retroverted or retroflexed. Are we right in scrupulously keeping women on their backs for nine days after confinement? Would not the gentle pressure of the enlarged womb by conterminous organs, supported by the varied play of the abdominal muscles, not only prevent the stagnation of decomposing fluids within the womb, but give it a better chance of growing to a proper shape and size than it does under the plan generally adupted in the treatment of puerperality? I think it likely, considering the good results obtained by Dr. Goodall in the treatment of 700 women at the Preston Retreat, near Philadelphia ; and a good idea of the institution, and of the practice there followed out, may be gathered from the following extract taken from Dr. Goodall's pamphlet :-

"The institution contains twenty beds, divided amongst four wards, five in each ward. The cubic capacity is about 1800 feet for each bed. About one hundred married women are delivered yearly. They are admitted, on an average, about sirteen days previously to confinement, and allowed to stay a month; they, however, generally only remain about eighteen days. The air admitted into the rooms is

\footnotetext{
- Transactions of the Obstetrical Society of London, vol. xri.
}

heated in the basement, and ventilation is maintained by a small jet of gas in the old-fashioned fireplace. Outside the hospital, puerperal fever was rife of late years. In Philadelphia and the city of New York, the mortality from puerperal causes (acknowledged as such) has been as high as one in forty-five, amongst all classes alike; if anything, more amongst the wealthier. The wards are used in rotation, one always being kept vacant for about two or three weeks. When a ward is emptied, the doors and windows are kept constantly opened until it is used again, and the whole of the walls, beds, furniture, and floors are scrubbed down with carbolised soap, and then mopped over with a weak solution of carbolic acid. No water is allowed to be used to the floors until the ward is emptied again. The nurses belonging to the ward go off duty for a week when it is closed, and go through a thorough system of purification. The beds are of straw, which are changed with each patient, the blankets and bedclothes being boiled in water with a small quantity of carbolic acid added. The feathers of the pillows and bolsters are only baked once a year, unless they should become soiled or have been used by a patient whose convalescence has been retarded. Every woman has a bath at least once a week before delivery. Any indication of enfeebled health is at once treated with quinine, steel, and phosphoric acid. Headaches and sleeplessness are dealt with by warm baths and large doses of bromide of potassium. The bowels are kept relaxed and purged. As soon as labour begins, the patient is placed in a warm bath. The membranes are generally ruptured artificially. The second stage is never allowed to be prolonged, the forceps or vectis being used......... Ergot is not given as an oxytocic, but as soon as the head comes to press on the perineum, a drachm is given. Should the perineum be torn, it is sewn up at once with silver sutures. A cylindrical compress is applied just above the fundus, and a tight binder applied for twelve hours, when it is removed, and not used again. The patients are confined on a deliverybed, and wheeled into tbe ward and removed to their beds. In not a single instance has flooding ever been caused by this muscular movement; if anything, it has rather tended to excite uterine contraction than otherwise. The next day after delivery the woman slips out and sits in a chair whilst her bed is made; this is repeated once or twice a day until the fourth or fifth day, at which time she may get up, dress herself, and do what she likes. No patient is forced to leave her bed, but the force of example is so great that most do. After-pains are immediately removed by quartergrain doses of morphia, given every hour until relief is obtained. If they be very obstinate, ten grains of quinine are given every six hours until the ears ring. It is an invaluable remedy in these cases. There are no bed-pans; vaginal injections are employed. Every woman washes herself daily with carbolised soap and a pad of fine oakum. No nurse, except for some special reason, is ever allowed to wash the woman's person. If the lochia be offensive, she is taken out of bed more frequently, and placed on the chair. Shnuld this not succeed, a vaginal injection is then used."

There is a strong presumption in favour of the belief that suckling has for one of its secondary purposes to stimulate the womb while in a transition state of structure. I have long ago confirmed Aran's assertion respecting the frequency of uterine disease in those who do not suckle, and this may be partly owing to defective involution in such women. Since I wrote on the subject, both Schroeder and Schneider aver that suckling promotes involution, and Serdukoff has satisfied himself tbat up to the first week of puerperality involution proceeds more rapidly in those who nurse than in those who do not. I am therefore among those who think the accoucheur should not leave the house without having seen the child applied to the mother's breast. Besides favouring involution by the singularly powerful effect of mammary irritation on the ovaries and womb, suckling also checks menstruation, and a few months' rest from menstrual congestion enables the womb to better recover its right size. The child will of course have to be weaned if he cannot thrive on the maternal milk; and, on the other hand, should the cervix be much ulcerated, suckling is very likely to prevent its cure by the usual surgical treatment, in which case also the child should be weaned.

What precedes relates to the prevention of defective involution. I pass over the most interesting part that it 
takes, during the first fortnight of puerperality, in the production of hæmorrhage, in the retention of decomposed fluids, in the production of puerperal metritis, lymphangitis, phlebitis, and fever, to direct attention to sundry morbid states that are to be observed a little later, that check involution by bringing on what is commonly called " $a$ bad getting up.'

There is always considerable debility caused either by length of labour, loss of blood, or nervous shock-debility requiring iron and quinine as well as wine and food. Everybody admits this requirement, and meets it more or less judiciously; but puerperal traumatism is little understood, and often completely unsuspected as the cause of a bad getting-up.

If a woman dies within forty-eight hours after delivery, the tissues of the womb will be found as black as ink. This bruising of the womb, the vagina, and subjacent tissue, is generally cured by nature; not so the fissures and rents of the cervix, simple or stellated, in weak and unhealthy subjects. I have already mentioned their frequency as admitted by the late Dr. Phillips and Dr. Atthill of Dublin; and the more frequent use of the forceps now advocated by the best men will inevitably increase the number of such lesions, for some will use instruments without being tho. roughly up to their management, and others will not always wait to use them till the os uteri is fully expanded. Thirty Jears of practice have made me certain of one thing-that each of these rents that refuse to heal of themselves may, by checking involution and in other ways, be the startingpoint of long years of uterine disease, often characterised by considerable viterine enlargement and hardness. With this conviction, I may be permitted to view with dismay any theory that would treat lengthily of uterine hardness and enlargement, in women who have borne children, and which did not take into consideration the frequent origin of such states in traumatic lesions of the cervix.

To check such lesions in their earliest stage, the best way is, after every case of labour, to suppose the existence of traumatism in the cervix and vagina, and to order vaginal injections to be made so soon as the lochia should cease to be red. I know of no better injection than a solution of acetate of lead, one drachm to the pint of tepid water. There is no likelihood of such an injection entering the uterus, and the chance of the occurrence of the uterine tormina that occasionally follow the use of injections, or the chance of some accidental awkwardness in the use of the instrument, is as notbing compared to the benefits generally derived from the practice. I recommend the practice as a matter of routine, without any previous examination of the patient, as not calculated to do harm, even if it be not wanted. If, three of four weeks after confinement, a bad getting up is notable for a vaginal discharge, bloody or muco-purulent, the patient should be carefully examined, and it will be found that uterine involution is being checked by some more serious lesion than congestion or subacute inflammation of the cervical mucous membranes. I refer to my Handbook of Uterine Therapeutics for the treatment of the chronic inflammatory lesions that grow out of puerperal traumatism.

The somewhat independent life and pathology of the neck and of the body of the womb is sometimes shown in the process of uterine involution, for the cervix may have nearly recovered its normal size and consistency, while the body may still remain large and flabby. Thus a large soft bag, being poised on a cervix already stiffened to its usual consistency, is ready to yield to the laws of gravitation and to bend at their point of junction in obedience to slight pressure. This is a very satisfactory explanation of postpartum flexions and versions, and it holds good to a great extent for those that follow abortion.

To explain these occurrences in the virgin womb, there is the over-weighting of the body of the womb, by repeated attacks of insufficiently relieved congestion or of inflammation, and in other cases the congenital imperfection of some part of the womb or of its ligaments; for, with regard to any considerable amount of softening of the virgin womb, in delicate young women, which has been lately asserted to be of not unfrequent occurrence, Dr. Henry Bennet, and some cther gynæcologists to whom I have spoken on the subject, are like me in not having met with such a condition. It follows from what $I$ have stated of the pcssible separate rate of involution of the cervix and of the body of the womb, that, supposing a considerable amount of cervical ulceration be found two or three months after parturition, this should not be taken as the sole lesion, for involution may be defective in the body of the womb, albeit complete in the cervix. There is no better way of promoting involution in such a case than by properly treating the cervical inflammation, and $I$ have already related a case in which the gradual involution of both body and cervix was brought about by the application of a strong solution of nitrate of silver, the cervix being without lesion. When the womb is found much too large several months after confinement and without cervical lesions, I order a ten-grain mercurial suppository to be put up the vagina, on going to bed, for the first fortnight, and afterwards on alternate nights, for two or three weeks, telling the patient to inject plain water in the morning, and a solution of alum and zinc at night. The exbibition of the mercury should be stopped if the gums show signs of impending salivation. In all cases of defective involution, with or without cervical inflammation, I give the usual doses of iron and strychnia, to act on the womb through the system, and also ergot, although it is difficult to understand that it can act as well on the womb. in a state of mutation as on the uterus in its ordinary state of nutrition-stability. When the womb is found large enough to admit a bougie to the depth of about four inches, and when this enlargement can be traced to a delivery that occurred a year or more previously, it is fair to attribute this enlargement to defective involution, but its treatment merges into that of one or more complications that will have certainly arisen in that time-displacements, internal metritis, hypertrophy, or ohronic inflammation of both body and neck of the womb. I shall say nothing of the treatment of such complicated cases, except that when defective involution is associated with an enlarged and hard cervix, lined with a diseased mucous membrane, nothing is better calculated to reduce the size of both the body and of the neck of the womb than an issue made in the most. accessible part of the cervix, by means of potassa fusa cum calce, the wound being freely swabbed with tincture of iron on alternate days for the following month. There should be no surgical treatment for the following three months, after which a second application of potassa fusa cum calce is often advisable.

Notwithstanding Dr. Serdukoff's assertion, the rate of uterine involution after pregnancy, at full term, can only be roughly guessed at, for the hand has to measure the womb through abdominal walls of variable degrees of thickness and laxity; but we know positively nothing respecting the rate of uterine involution after abortion, and it would be well if the structure of the uterine walls were examined with the microscope when women die after abortion in our hospitals. From the great frequency of uterine disease after abortion, and from the fact of its being contrary to order that the womb should be called upon to undergo fatty degeneration, unless after pregnancy, at full term, it is presumable that the two facts are related as cause and effect. Defective involution is probably the reason why one abortion is so of ten followed by others, the uterine walle refusing to properly expand for the safe guardianship of the fotus, whereas, if the body of the womb be healthy, pregnancy will of ten proceed satisfactorily, in spite of serious cervical inflammatory lesions. It is, of course, still possible for pregnancy to be brought to a satisfactory issue under the most unfavourable circumstances; for, in a case now under observation, the cervix was extensively torn in a first labour eighteen years ago, and abortion was repeated seven times consecutively. An enlarged body, as well as a considerable amount of cervical enlargement and inflammation, was recognised some years ago by Dr. George Bird, but the treatment he recommended was not carried out. From the symptoms complained of previous to the eighth conception, it is clear that the uterine disease had not abated, and yet this lady went her full time and was safely delivered of a fine boy. During the following four years she did:not conceive, long periods of aggravated dysmenorrhoaa and menorrhagia being separated by ten days of miserable health. I found the body of the womb about three times its usual size, the cervix similarly enlarged, very hard and sensitive, the os uteri irregularly shaped, and its mucous membrane of a dusky red.

To prevent the defective involution that follows abortion, this should be treated like an aoute disease of the womb. 
The patient should rest in bed, so long as pelvic pains are severe, and warm linseed-meal poultices should be applied to the abdomen. Linseed-tea or poppy-head injections are very useful during the first week, and then injections made by adding a drachm of laudanum and of acetate of lead to a pint of tepid water, twice a day. When the patient resumes her usual mode of life, if there be back pain or bearingdown pains on slight exertion, a vaginal discharge, staining the linen yellow, and debility in spite of tonic treatment, then the patient should be examined, and surgical applications should be made to the ulcerated cervix. Involution is so often defective after abortion because women get about too soon, and too soon resume matrimonial intercourse, and the want of some definite understanding respecting the rules to be adopted after abortion renders it a fertile source of disease. Much of this would be prevented if we could persuade the public that a month's treatment is not too much for an ordinary miscarriage at the third or fourth month, and that a bad miscarriage is worse than a confinement.

It is fair to promise a woman that defective uterine involution, become permanent, may be cured by a subsequent pregnancy. I have seen cases, and others are on record, in which uterine fibroids of considerable size have been softened and swept away by the flood-tide of pelvic circulation that characterises pregnancy and puerperality; so it is easy to understand that tissues, by defective involution long made permanently too hard, may soften, allow of a perfect pregnancy and of the subsequent building up of a new womb after a better model. I have seen instances of this and other cases in which, by a judicious management of puerperality, the womb assumed its proper shape instead of being extensively retroverted, as it had previously been for years.

Seymour-street, Great Camberland-place.

\section{NOT E S}

ox

\section{SOME PATHOLOGICAL FEATURES OF STRANGULATED HERNIA,}

BSPECIALLY WITH REFERENCE TO THE CAUSE OF DEATH, AND TO AN EXPLANATION OF SO-CALLED STRANGU LATED OMENTUM.

\section{BY RUSHTON PARKER, F.R.C.S., M.B, PATHOLOGIST TO THE ROYAI INBIRMARY,} SURGEON TO THE STANIFY HOSPITAL, LIVERPOOL.

A REVIEW of some of the cases of patients who have died after strangulated hernia, in which $I$ have examined the post-mortem appearances, and of other cases which I have recently seen, brings with it an answer, more or less satisfactory, to the following questions:-1. Are the anatomical appearances adequate to account for persistence of the symptoms of strangulation, and so for death? and if so, what explanation must be placed upon them? 2. Is strangulated omentum alone, as such, to be accepted as a form of strangulated hernia?

Three of these cases have occurred in the Liverpool Infirmary; two I have had access to in the practice of other professional friends in the town. To all these gentlemen I am indebted for permission to make use of their materials. The only case that recovered was one of the last two, and is introduced here as illustrating a point to which another case pointedly alludes. A sixth case occurred in my own hospital practice.

CASE 1.-A man, aged thirty-nine, with left inguinal and scrotal hernia of thirty years' standing, formerly all reducible, latterly not. On Nov.9th strangulation, reduction in infirmary by taxis after twelve hours; omentum left in sac; uncertainty whether any bowel yet remained. Symptoms returned. Herniotomy by Mr. Harrison after two days, sac being opened; no gut found; much omentum reduced; some left in sac fixed by fibrous adhesions. Death in thirty-six hours. The post-mortem examination revealed sticky peritonitis; transverse colon dragged tightly down to the level of the umbilicus by omentum; above this point intestines distended, below contracted; bit of ileum near lower end, showing where it had been strangulated, dark purple, probably recoverable, lying near internal ring.

CASE 2.-A man, aged sixty-four, with a left inguinal hernia of a few weeks' standing, strangulated a couple of days. Herniotomy, at the infirmary, by Mr. Harrison; sac not opened, and the bowel reduced by simply bolding up the sac, the neck of which bad been bent on itself. There was no stricture. Symptoms returned in twenty-four hours, and death took place in four days. The post-mortem examination revealed stick peritonitis and moderate distension above the piece of ileum which had been inslicated, and which was eight feet from lower end; the end of the omentum was lying loosely in the sac, and was easily withdrawn. No narrowness of sac's neck.

Why did these cases die? All direct cause of strangulation had been removed, but what maintained the symptoms? It would seem that the injured bowel, becoming adherent by peritoneal lymph to the adjacent wall or viscus, and its muscular coat having become paralysed above the strangulated portion, an amount of obstruction was effected which would, under ordinary circumstances, be impracticable. Mr. Bryant* refers to a case which, in several points, resembles Case 1, and in which obstruction of the transverse colon and symptoms of strangulation were kept up by adhesion and traction of omentum. I am disposed to regard the very slightest adhesion and folding of a previously strangulated bowel as capable of effecting fatal obstruction when combined with paralysis of the muscular coat above. This last feature is referred to by Sir James Paget in his Clinical Lectures, 1872. This is also the explanation I have to offer in the following unsuccessful instance.

CASE 3.-In March, 1875, a woman, aged fifty-five, was admitted into the Stanley Hospital, suffering from a right femoral hernia of four days' duration, during which time she had suffered from symptoms of strangulation. I performed herniotomy at once, and opened the sac, which contained a small spherical piece of ileum, only part of the circumference of the gut. The bowel was severely nipped and furrowed, and had a white patch on it, but was returned. The symptoms never abated, and death took place in about thirty-six hours. The post-mortem examination revealed sticky peritonitis, the injured piece of gut adhering to abdominal wall near the crural ring; bowel distended above, contracted below this point. Here, too, though the symptoms persisted, no actual strangulation remained, but obstruction was nevertheless, I believe, kept up by the adbesion of the injured bowel in a folded state, aided by the paralysis of the bowel above.

In each of these cases, though strangulation was removed, still obstruction remained-an amount of obstruction which would probably be imperative in the case of an uninjured bowel.

Another feature of Case 1 attracts attention. If this case had recovered, it might have been described, with some show of reason, as a case of "strangulated omental hernia." It was noticed that at the post-mortem examination the bowel was distended as far as the middle of the transverse colon, which was dragged down by the colon engaged in the inguinal canal and scrotum and by that loosely adherent to the neighbourhood of the internal ring. But there had certainly in this hernia been a piece of ileum which was reduced by taxis; the redundant omentum was reduced at the herniotomy, and both took up a position close to the internal ring. By this time, however, no doubt the distended bowel was paralysed; before it could recover its power, the omentum was firmly enough adherent below to keep up its slight drag on the colon; hence, the continuance of the obstruction.

In reference to the supposed strangulated omental hernia, let me here quote a passage out of Lawrence on Ruptures, fifth edition, p. 454: "If strangulation of the omentum by the ring is sufficient to produce dangerous and mortal consequences, must they not be equally expected from that stricture which is eaused by the ligature?" Here the ligature of the omentum is condemned on the supposition-a most reasonable one-that straugulation must be a consequence. But has not ligature of the omentum been over and over again praetised without harm? 